\title{
Molecular Diversity Analysis and Cultivar Identification using Simple Sequence Repeat (SSR) Markers in Soybean [Glycine max (L.) Merrill]
}

\author{
Shipra Deo', Anto James ${ }^{1}$, Sanjeev Kumar Singh ${ }^{1}$, \\ C. B. Singh ${ }^{2}$ and Mukesh Kumar Rana ${ }^{1} *$ \\ ${ }^{1}$ ICAR-National Bureau of Plant Genetic Resources, \\ Pusa Campus, New Delhi 110 012, India \\ ${ }^{2}$ Department of Botany, Tilka Manjhi Bhagalpur University, \\ Bhagalpur, Bihar 812 007, India \\ *Corresponding author
}

\section{A B S T R A C T}

\section{Keywords}

Genetic diversity, Glycine max, Molecular markers, Microsatellite markers, Cultivar identification

\section{Article Info}

Accepted: 07 March 2020 Available Online: 10 April 2020
Soybean [Glycine max (L.) Merrill] is ranked number one among major oil crops and is the second most important oil seed crop of India. There has been significant growth in both area under cultivation and production, however, it still has lower productivity. In the present study, use of SSR markers has been made to study genetic diversity in the Indian soybean cultivars and for their identification. Ninety-six soybean cultivars were used and genetic diversity was assessed using 49 microsatellite markers distributed across the soybean genome. A total of 230 bands were amplified of which 229 bands were found to be polymorphic. The number of amplified bands ranged from 2 to 7 with an average value of 4.73 . The PIC values of the primers ranged from 0.13 to 0.77 with an average of 0.61 . The heterozygosity index values varied between 0.14 to 0.80 with an average of 0.66 . The genetic similarity values in pair-wise comparison of cultivars ranged from 0.59 to 0.94 with an average similarity index of 0.71 . Based on cluster analysis, soybean cultivars included in the study were grouped into two major clusters. However, all cultivars could be grouped into eight clusters. Among these eight clusters, cluster $\mathrm{V}$ was found to be the largest with 33 cultivars followed by cluster IV (30 cultivars), cluster I (16 cultivars), cluster III (4 cultivars), cluster VIII (03 cultivars) and cluster II ( 2 cultivars). Unique bands, present in one particular cultivar only and absent in all other cultivars, were observed for seven cultivars namely, Lee, MACS-58 KHsb-2 JS-97-52 PK-471, Bragg and Co-3. Six primers produced these seven unique bands with primer Gly SATT-586 producing two unique bands of sizes 170bp and 230bp in cultivars Lee and MACS-58, respectively. All the cultivars could be unequivocally discriminated from one another using SSR marker set included in the study.

\section{Introduction}

Soybean [Glycine $\max ($ L.) Merrill] is an important leguminous crop having 40-44\% protein and 20\% oil (Singh et al., 1999). Due to its versatile nutritional qualities and multiple uses, soybean is also known as the golden bean or miracle bean or wonder crop (Gopinath et al., 2015). In the international world trade, soybean is ranked number one among major oil crops such as rapeseed, groundnut, cotton seed, sunflower, linseed, sesame and safflower (Chung and Singh, 2008). Soybean is the second most important 
oil seed crop of India, next to mustard. Although soybean has made significant strides for both area under cultivation and total production, it still suffers as far as productivity is concerned, particularly pertaining to climate, edaphic, production, and technology aspects that hinder its higher productivity. One way to enhance productivity is to enhance gains through genetic improvement, whereby to ascertain the existing levels of variability and quantifying genetic diversity is essential.

Genetic diversity in soybean has been assessed using various types of marker systems viz., morphological, biochemical, cytological and molecular including DNA markers. Morphological and biochemical markers are limited in number, stage-specific and are highly influenced by the environmental conditions. Different types of DNA markers like RFLP (Botstein et al., 1980), RAPD (Williams et al., 1990), ISSR (Zietkiewicz et al., 1994), AFLP (Vos et al., 1995) and microsatellite or SSR (Tautz and Renz, 1984) have been utilized for genetic diversity analysis at molecular (DNA) level in crop plants. DNA markers are more informative, stable and reliable, and hence are a powerful tool for diversity analysis and cultivar identification. Among different DNA marker types, Simple Sequence Repeat (SSR) or commonly called microsatellites are an excellent system. These SSR's can be mono-, di-, tri-, tetra- or penta-nucleotides with different lengths of repeats motifs and are abundant throughout the eukaryotic genome (Powell et al., 1996). Over other techniques, SSRs have many advantages such as rapid and reliable detection, abundance in the genome, co-dominant inheritance, reveal high heterozygosity and high levels of polymorphism with high reproducibility (Akkaya et al., 1995, Lacape et al., 2007). The hypervariable number of repeat units makes microsatellite markers an excellent tool for genotype differentiation and in evaluation of genetic diversity. High level of polymorphism at the SSR loci has been reported in soybean by various workers (Bisen et al., 2015; Rani et al., 2016; Nawaz et al., 2017) and these microsatellite markers have been extensively used in genetic diversity analysis and in DNA fingerprinting of soybean genotypes as well (Ghosh et al., 2014; Chauhan et al., 2015; Pagar et al., 2017; Gupta and Manjaya, 2017; Zhao et al., 2018, Koutu et al., 2019). The use of SSR markers has been made in the present study with the aim to prove the efficiency of microsatellite markers in evaluating genetic diversity of Indian soybean cultivars and for their molecular identification.

\section{Materials and Methods}

\section{Plant material and DNA extraction}

Ninety-six different cultivars of soybean released for different agro- climatic regions of the country over different years were selected for the present study. The details of the cultivars including pedigree, cultivation zone, year of release and breeding centre of the varietal material used in the study are provided in Table 1. Eighty-two SSR primer pairs selected from the 20 linkage groups of soybean from the previous study (Song et al., 2004) were initially screened in this study to find the ones giving sharp, scorable and polymorphic amplification products. The primer sequences with their linkage group location are available at https://www. soybean.org /d/page/\# soybean data base.

The seeds of soybean cultivars used in the study were sown in plastic pots in the green house at ICAR-National Bureau of Plant Genetic Resources (NBPGR), New Delhi. Five gram of leaf tissue from fifteen-days old seedlings was harvested in bulk from each cultivar for genomic DNA extraction. 
Genomic DNA was extracted following CTAB (Cetyl-trimethyl-ammonium bromide) method as described by Saghai-Maroof et al., (1984) with minor modifications. The concentration and quality of the isolated genomic DNA was estimated using the Nanodrop spectrophotometer (ND-1000, USA) and a final working concentration of $10 \mathrm{ng} / \mu \mathrm{L}$ was made and stored at $-4^{\circ} \mathrm{C}$ for further analysis.

\section{PCR amplification of SSR markers}

Five different cultivars of soybean namely, Palam Soya, Harit Soya, Phule Kalyani, Improved Pelican and Alankar were used to screen the SSR primers. After screening of all the primers on this panel of five cultivars, 49 primers were finally selected for further study on the basis of clear polymorphic bands and good reproducibility (Table 2 ).

The reaction mixture $(25 \mu \mathrm{L})$ consisted of 3.5ng genomic DNA, $2.5 \mu \mathrm{L}$ of $10 \mathrm{X}$ PCR Buffer (10Mm Tris-HCL, pH-8.3 and 50mM KCL). $0.2 \mathrm{mM}$ dNTPs mix, $0.8 \mu \mathrm{M}$ SSR primers (forward and reverse), $3 \mathrm{mM} \mathrm{MgCl}_{2}$ and $0.5 \mu \mathrm{L}$ Taq DNA polymerase.

PCR amplification cycle was optimized at different temperatures and observed for sharp bands. PCR amplification consisted of initial denaturation at $95^{\circ} \mathrm{C}$ for $5 \mathrm{~min}$, followed by 40 cycles of $1 \mathrm{~min}$ at $94^{\circ} \mathrm{C}, 1 \mathrm{~min}$ at $55^{\circ} \mathrm{C}, 1$ min at $72^{\circ} \mathrm{C}$ and final extension at $72^{\circ} \mathrm{C}$ for 8 min. All PCR reactions were carried out in thermal cycler from M/S MJ Research (Model PTC 200). At the time of electrophoresis PCR products were mixed with $1 \mu l$ of gel loading dye (6x buffer: bromophenol blue, 0.25; xylene cyanol FF, 0.25; and glycerol in water $30 \%$ ) and spun briefly in a microfuge before loading on to the gel.

The amplification products were electrophoresed and visualized on a $3 \%$ metaphor-agarose gel stained with ethidium bromide. The size of the amplified SSR fragments was estimated by running $100 \mathrm{bp}$ DNA ladder (M/S BR Biochem Life Sciences) in the gel as a standard size marker. After electrophoresis the gel was photographed under a UV light in a gel documentation system.

\section{SSR data scoring and analysis}

Amplified fragments of different sizes were considered as different alleles. Only distinct and reproducible bands were scored as present (1) or absent (0) for each SSR primer pair. This scored data was recorded in an MS excel spread sheet and the resultant data matrix was subjected to further analysis.

Various band statistics were calculated which consisted of total number of bands, total number of polymorphic bands, percent polymorphism, number of bands per marker, allelic frequency, unique alleles, gene diversity, heterozygosity, polymorphic information content (PIC) [Botstein et al., (1980)], Effective multiplex ratio (EMR), Resolving Power (RP), and Marker Index (MI). Marker Index (MI), the measure of marker utility was obtained as a product of PIC and Effective Multiplex Ratio and was calculated in accordance with Powell et al., (1996).

Resolving Power (RP) of each primer pair was calculated as per Prevost and Wilkinson (1999). Resolving Power of a marker gives us an indication of the discriminatory potential of the marker. The data analysis was carried out using computer software NTSYS-pc version 2.2 (Rohlf, 2009) for calculating Simple Matching coefficient values and to generate a dendrogram to decipher genetic relationship among the genotypes. Principal co-ordinate analysis (PCoA) was also computed using the same NTSYS-pc software. 


\section{Results and Discussion}

Genetic diversity assessment and cultivar identification is important for crop improvement management and protection of genetic variability (Chandra et al., 2013). DNA markers nowadays are routinely used in such studies to provide authenticity to morphological markers and to arrive at accurate estimates of genetic relationships among genotypes. Moreover, DNA markers, particularly SSR markers, have been the preferred choice in breeding programs as these are more authentic and less influenced by environmental fluctuations (Vinu et al., 2013). Several previous investigators recommended the use of microsatellite markers as a rapid and powerful tool for genetic diversity assessment in soybean and different plant species.

\section{SSR polymorphism survey}

In the present investigation 82 SSR primers were initially chosen based on published research work (Song et al., 2004). Out of these 82 primers, 49 SSR primers were able to amplify the genomic DNA of soybean and were informative in discriminating the 96 soybean cultivars. The representative amplification profile of 96 soybean cultivars using SSR primer SATT-268 is shown in Figure 1.

A total of 230 bands were amplified by the 49 SSR primers, out of which 229 bands were found to be polymorphic and only one band was monomorphic which was by using the primer SATT-346 (Table 3). Forty-eight primers produced $100 \%$ polymorphic bands, while one primer SATT-346 produced $80 \%$ polymorphic bands. The percentage of polymorphism produced by SSR primers was, hence, found to be $99.59 \%$. Further, it was observed that there was no correlation between per cent polymorphism and PIC values as SSR primer Sat-366 showed minimum PIC values but was 100 per cent polymorphic. The average percentage of polymorphism produced by the 49 SSR primer in our study was found to quite high compared to previous studies (Kumar et al., 2009; Singh et al., 2010; Bisen et al., 2015) which might be due to inclusion of genetically more diverse material in our study.

The number of alleles amplified by each primer pair ranged from 2 to 7 with an average value of 4.73 (Table 3). The SSR primers Gly Satt-586 and Satt-038 each, generated the maximum number of seven alleles while the primer Sat-366 produced the minimum number of alleles which was two. The product size of the bands ranged from 80bp (Satt-038) to 480bp (Gly Satt-577).

The allele frequency obtained in earlier studies was quite similar to what we observed in our study (2 to 7 alleles) per locus. For example, Yun-Lai et al., (2009) detected 2 to 7 alleles per locus in 83 soybean cultivars with 43 SSR primers. A similar result was reported by Chauhan et al., (2015) using 21 SSR markers in 48 Indian Soybean cultivars. The average value of alleles per locus (4.73) detected in our study was found to be similar with that observed by Tantasawat et al., (2011) (4.8 allele/locus).

The PIC values of the primers ranged from 0.13 to 0.77 with an average of 0.61 (Table 3). The maximum PIC value was recorded for the primer Gly SATT-449, whereas the minimum value was recorded for primer Sat366. Tantasawat et al., (2011) found the polymorphic information content (PIC) among 25 soybean genotypes to be ranging from 0.13 to 0.88 with an average of 0.60 . The results of this study were comparable to what we had observed in our study, with regards to polymorphic information content (PIC) and average number of alleles per 
locus. Ghosh et al., (2014) observed PIC among 32 soybean cultivars varies from 0.21 (S26) to 0.83 (S27) with an average of 0.51 , which was quite low compared to what we have observed in our study. The heterozygosity index values ranged from 0.14 (Sat-366) to 0.80 (Gly SATT-449) with an average of 0.66 (Table 3 ).

The genetic similarity values (Simple Matching) ranged from 0.59 to 0.94 with an average similarity index of 0.71 (similarity matrix table not shown). The lowest average similarity coefficient value (0.59) was shown by the cultivar Hardee with Pant soybean and JS-71-05. The highest similarity coefficient value (0.94) was shown by MAUS-81 and Paratap Soya. The Marker Index (MI) values ranged from 1.348 to 5.082 with an average value of 3.30. Resolving Power values ranged from 2 to 2.23 with an average of 2.0 for the polymorphic markers.

The discriminatory power of SSR markers for varietal differentiation has been observed in earlier studies also. The gene diversity in our study ranged from 0.44 (Satt-243) to 0.80 (Gly SATT-449) with an average of 0.69. Diwan and Cregan (1997) reported a relatively large mean gene diversity of 0.80 , which was much greater than that observed in our study.

Similarly, Cho et al., (2006) reported the genetic diversity to range from 0.41 to 0.93 with an average of 0.69 , which was quite similar to our findings. Similarly, Chotiyarnwong et al., (2007) reports the mean gene diversity of the soybean cultivars to be 0.831, which is quite high compared to our study. Yoon et al., (2009) reports similar levels of genetic diversity that range from 0.35 to 0.94 with an average value of 0.70 . Nawaz et al., (2017) reports a relatively low average genetic diversity of 0.4 in 100 Korean wild soybean accessions, using 42 SSR markers, this was found to be far lesser than what was observed in our study.

The results of the present investigation are in disagreement with others with respect to SSR polymorphism surveys. For example, the study of Velusamy et al., (2013) reported the number of alleles to range from 6 to 11 alleles, with a mean value of 9.11 per locus and the total number of alleles to be 82 in 178 wild soybean accessions using nine SSR markers. The discrepancy in the observations might be due to very less number of primers used in their study.

Kumawat et al., (2014) assessed the genetic diversity in 82 soybean accessions using 40 SSR markers and observed, unlike in our study, the number of alleles for SSR loci to range from 2 to 4 with an average of 2.97 alleles per marker. PIC values in their study ranged from 0.10 to 0.74 with an average of 0.48. Rani et al., (2016) characterized 102 Indian soybean cultivars using 10 SSR primers only and observed PIC values to range from 0.47 to 0.81 and with a total 50 alleles.

\section{Genetic diversity and relationships among cultivars}

A cluster analysis of all the cultivars included in the study was carried out based on the similarity indices which were derived from 49 SSR primers. Based on cluster analysis, soybean cultivars were grouped into two major clusters i.e. A and B (Figure 2).

However, all cultivars could be grouped into total eight clusters. Among these eight clusters, cluster $\mathrm{V}$ was found to be the largest with 33 cultivars followed by cluster IV (30 cultivars), cluster I (16 cultivars), cluster III (4 cultivars), cluster VIII (03 cultivars) and cluster II (2 cultivars). Cultivars Pratap Soya and MAUS-81, although from different 
sources and with different pedigrees, shared the maximum similarity based on the chosen set of SSR markers. However, cultivars JS9305 and JS-9560 were also found to be genetically similar based on the clustering.

These two cultivars were from the same breeding center i.e. JNKVV Jabalpur and were found to share a common pedigree of secondary selection from PS-73-22. There was no discernible clear pattern in grouping of cultivars into clusters either year-wise or from different breeding centers or zones. Loose packaging of cultivars into different clusters was conspicuous with respect to cultivars from VPKAS, Almora in cluster I, MACS, Pune in cluster IV and from GBPUA \& T, Pant Nagar in cluster V.

Cultivars sharing common pedigree also tended to remain together within the same cluster. For example, cultivars MACS-57, MACS-58, and MACS-124 sharing common pedigree JS2 X Improved Pelican were found to be in cluster IV. Cultivars Ahilya-1(NRC2) and Ahilya-2 (NRC-12) grouped in cluster $\mathrm{V}$ have a common parentage. Similarly, four cultivars viz., Hara soya, MACS-450, PS1042 and Pusa 98-14 grouped in cluster V has a common parent 'Bragg', and similar finding have been observed by Bisen et al., 2015. In clustering, the genotypes with similar pedigree were grouped together.

In earlier studies also, grouping of Indian soybean genotypes into two (Chotiyarnwong et al., 2007, Ghosh et al., 2014, Bisen et al., 2015; Chauhan et al., 2015, Wang et al., 2015, Koutu et al., 2019) and three clusters (Kumawat et al., 2014, Gupta and Manjaya, 2017) have been reported, indicating the close relatedness among cultivars. The cluster analysis shows that the cultivars which are quite distinct from the rest might be source of novel genes/ allels and can be utilized in soybean breeding programs. Principal Coordinates analysis (PCoA) results (not shown) corroborated the finding of cluster analysis strengthening the clustering of cultivars into different groups.

\section{Soybean cultivar identification}

Cluster analysis shows that all the cultivars could be discriminated from one another using the set of 230 markers from 49 primers (Figure 2). These findings are useful to establish the identity of cultivars in the court of law in case any dispute with respect to cultivar identification arises.

Presence of unique bands helped in the identification of specific genotypes and is useful for DNA profiling of cultivars. In the present study, we observed unique bands, present in one particular cultivar only and absent in all other cultivars, for seven cultivars namely, Lee, MACS-58 KHsb-2 JS97-52 PK-471, Bragg and Co-3.

Six primers produced these seven unique bands with primer Gly SATT-586 producing two unique bands of sizes 170bp and 230bp in cultivars Lee and MACS-58, respectively (Table 3). Similar findings have been reported earlier by Tantasawat et al., (2011) and Sahu et al., (2012) who reported seven unique alleles in their study and Koutu et al., (2019) obtained eight unique alleles in soybean genotypes using SSR primers.

Kumawat et al., (2014) reported five unique alleles in a set of 82 soybean genotypes, whereas Rani et al., (2016) reported four unique allele in a set of 102 Indian soybean cultivars in their study. These unique alleles can be utilized for the identification of soybean cultivars and for isolating distinguishing genes/alleles for further studies. 
Table.1 List of Indian soybean cultivars [Glycine max (L.) Merrill] along with their pedigree, zone, year of release and releasing centre

\begin{tabular}{|c|c|c|c|c|c|}
\hline $\begin{array}{l}\text { S. } \\
\text { No. }\end{array}$ & Cultivar & Pedigree & Zone & $\begin{array}{l}\text { Year of } \\
\text { release }\end{array}$ & Releasing Centre \\
\hline 1 & JS 335 & JS78-77 × JS71 -5 & $\mathrm{CZ}$ & 1994 & RVSKVV, Sehore (MP) \\
\hline 2 & Palam soya & JS72-451xPunjab-1 & NHZ & 2005 & CSKHPKV, Palampur (HP) \\
\hline 3 & JS 9560 & Secondary selection from PS 73-22 & $\mathrm{CZ}$ & 2006 & JNKVV, Jabalpur (MP) \\
\hline 4 & JS-9305 & Secondary selection from PS 73-22 & $\mathrm{CZ}$ & 2002 & JNKVV, Jabalpur (MP) \\
\hline 5 & Harit Soya & Himso $1520 \times$ Bragg & $\mathrm{NHZ}$ & 2001 & CSKHPKV, Palampur- (HP) \\
\hline 6 & VL Soya-2 & Selection from VHC 856007 & NHZ & 1989 & VPKAS, Almora (UP) \\
\hline 7 & VL Soya-21 & Selection from VHC 3055 & NHZ & 1996 & VPKAS, Almora (UP) \\
\hline 8 & VL Soya-59 & $(\mathrm{Pb} 1 \times \mathrm{VLS} 2) \times \mathrm{EC} 361336$ & NHZ & 2008 & VPKAS, Almora (UP) \\
\hline 9 & VL Soya-63 & VLS2 $\times($ Bragg $\times$ VHC3022 $)$ & NHZ & 2008 & VPKAS, Almora (UP) \\
\hline 10 & VL Soya-47 & Selection from KHSF- 3-1-1 & NHZ & 2000 & VPKAS, Almora (UP) \\
\hline 11 & VL Soya-1 & Mutant of Bragg & NHZ & 1985 & VPKAS, Almora (UP) \\
\hline 12 & VL Soya-65 & Selection from local cultivar & NHZ & 2010 & VPKAS, Almora (UP) \\
\hline 13 & RVS-2001-4 & JS93-01 × EC390981 & $\mathrm{CZ}$ & 2014 & RVSKVV, Gwalior \\
\hline 14 & Phule Kalyani & JS335 × Ankur & SZ & 2006 & MPKV, Rahuri \\
\hline 15 & Kalitur & Indigenous native cultivar & $\mathrm{CZ}$ & 1969 & JNKVV, Jabalpur (MP) \\
\hline 16 & Lee & $\mathrm{S}-100 \times \mathrm{CNS}$ & NHZ & 1975 & VPKAS, Almora ((UP) \\
\hline 17 & Hardee & D 49-772 $\times$ Improved Pelican & $\mathrm{SZ}$ & 1976 & GKVK, Bangalore \\
\hline 18 & Improved Pelican & Tanloxi × PI60406 & SZ & 1969 & VNMKV, Parbhani \\
\hline 19 & Ankur & SPS from composite of 22 crosses & NPZ & 1976 & IARI, New Delhi \\
\hline 20 & Type 49 & Selection from indigenous material & $\mathrm{CZ}$ & 1978 & JNKVV, Jabalpur (MP) \\
\hline 21 & Punjab 1 & Selection from Nanking cultivar & NPZ & 1978 & PAU, Ludhiana \\
\hline 22 & Bragg & Jackson $\times$ D 49-2491 & $\mathrm{CZ}$ & 1978 & ICAR, Indore \\
\hline 23 & Alankar & D 63-6094 × D61-4249 & NPZ & 1978 & IARI, New Delhi \\
\hline 24 & KHSb-2 & Manloxi × EC 39821 & SZ & 1982 & GKVK, Bangalore \\
\hline 25 & Shilajeet & Selection from EC 9309 & NHZ & 1980 & VPKAS, Almora (UP) \\
\hline 26 & Pusa 40 & $8-3 \times$ Lee & NPZ & 1981 & IARI, New Delhi \\
\hline 27 & Js-2 & Selection from Tehri Garhwal material. & $\mathrm{CZ}$ & 1982 & JNKVV, Jabalpur (MP) \\
\hline 28 & Gaurav (JS 72-44) & D 60-9647 × EC 7034 & $\mathrm{CZ}$ & 1982 & JNKVV, Jabalpur (MP) \\
\hline 29 & Durga (JS 72-280) & EC14437 × Bragg & $\mathrm{CZ}$ & 1982 & JNKVV, Jabalpur (MP) \\
\hline 30 & Co- 1 & Selection from EC398321 & SZ & 1982 & TNAU, Coimbatore \\
\hline 31 & Pusa-22 & Punjab $1 \times$ Clark 63 & NPZ & 1983 & IARI, New Delhi \\
\hline 32 & $\begin{array}{l}\text { Gujarat(J-202) } \\
\text { soyabean -2 }\end{array}$ & Selection from Geduld cultivar & SZ & 1983 & MAUS, Parbhani \\
\hline 33 & $\begin{array}{l}\text { Gujarat(J-231) } \\
\text { Soyabean } 1\end{array}$ & Selection from Punjab-1 & $\mathrm{SZ}$ & 1983 & MAUS, Parbhani \\
\hline 34 & PK 327 & UPSM $82 \times$ Semmes & NPZ & 1983 & GBPUA \& T, Pant Nagar \\
\hline 35 & PK 262 & UPSM97 $\times$ Hardee & NPZ & 1983 & GBPUA \& T Pant Nagar \\
\hline 36 & Birsa soybean -1 & Spontanous mutant of 'Sepaya Black' & NPZ & 1983 & Ranchi \\
\hline 37 & JS-76-205 & Kalitur $\times$ Bragg & $\mathrm{CZ}$ & 1990 & JNKVV, Jabalpur (MP) \\
\hline 38 & Pusa-37 & Bragg $\times$ Java 16 & NPZ & 1985 & IARI, New Delhi \\
\hline 39 & PK-308 & T $31 \times$ Hardee & NPZ & 1985 & GBPUA \& T, Pant Nagar \\
\hline 40 & Monetta & Exotic cultivar EC 2587 & SZ & 1985 & MACS, Pune \\
\hline
\end{tabular}




\begin{tabular}{|c|c|c|c|c|c|}
\hline 41 & MACS-13 & Hampton $\times$ EC 7034 & $\mathrm{SZ}$ & 1985 & MACS, Pune \\
\hline 42 & PK-472 & Hardee $\times$ Punjab-1 & NPZ & 1986 & GBPUA \& T, Pant Nagar \\
\hline 43 & PK-416 & UPSM $534 \times$ S 38 & NPZ & 1986 & GBPUA \& T, Pant Nagar \\
\hline 44 & Shivalik & Selection from segregating PK 73-55 & NHZ & 1990 & CSKHPKV, Palampur- (HP) \\
\hline 45 & Pusa-24 & Shelby $\times$ Bragg & NPZ & 1987 & IARI, New Delhi \\
\hline 46 & Pusa-16 & CNS $\times$ Lee & NPZ & 1987 & IARI, New Delhi \\
\hline 47 & JS-75- 46 & Improved Palican x Semmes & $\mathrm{CZ}$ & 1987 & JKVV, Jabalpur (MP) \\
\hline 48 & Pusa-20 & Bragg $\times$ Lee & NPZ & 1988 & IARI, New Delhi \\
\hline 49 & MACS-58 & JS2 $\times$ Improved Pelican & $\mathrm{SZ}$ & 1989 & MACS, Pune \\
\hline 50 & AD-1(UGM-33) & Selection from "Hill cultivar" & SZ & 1990 & TNAU, Coimbatore \\
\hline 51 & Pant soybean 564 & (UPSM $534 \times$ Ankur) $\times$ Bragg & NPZ & 1991 & GBPUA \& T, Pant Nagar \\
\hline 52 & JS-80-21 & JS75-1 × PK 73-94 & $\mathrm{CZ}$ & 1991 & JANKVV, Jabalpur (MP) \\
\hline 53 & JS-71-05 & Selection from Lee type exotic material & $\mathrm{CZ}$ & 1991 & JANKVV, Jabalpur (MP) \\
\hline 54 & MACS-124 & JS $2 \times$ Improve Pelican & SZ & 1992 & MACS, Pune \\
\hline 55 & MACS-57 & JS2 $\times$ Improved Pelican & SZ & 1992 & MACS, Pune \\
\hline 56 & JS-79-81 & Bragg $\times$ Harsoy-Deciduous & SZ & 1994 & IASRI \\
\hline 57 & Aarti (MAUS 1) & Mutant from DS 87-14 & SZ & 1996 & MAUS, Parbhani \\
\hline 58 & SL-295 & PK416 × PK564 & NPZ & 1997 & PAU, Ludhiana \\
\hline 59 & Sneh (KB 79) & Hardee $\times$ Monetta & SZ & 1997 & GKVK, Bangalore \\
\hline 60 & Pooja (MAUS 2) & Selection from SH 84-14 & SZ & 2014 & MAUS, Parbhani \\
\hline 61 & Pant soybean 1042 & Bragg $\times$ PK 416 & NPZ & 1997 & GBPUA \& T, Pant Nagar (UP) \\
\hline 62 & Pant soybean 1029 & PK $262 \times$ PK317 & NPZ & 1997 & GBPUA \& T, Pant Nagar \\
\hline 63 & Pant soybean 1024 & PK $308 \times$ PK317 & NPZ & 1997 & GBPUA \& T, Pant Nagar \\
\hline 64 & Co- Soya -2 & UGM21 × JS335 & SZ & 1995 & TNAU,Coimbatore \\
\hline 65 & Ahilya-1 (NCR-2) & Induced mutant of Bragg & $\mathrm{CZ}$ & 1996 & ICAR, Indore \\
\hline 66 & Ahilya-2(NCR -12) & Induced mutant of Bragg & $\mathrm{CZ}$ & 1996 & ICAR, Indore \\
\hline 67 & Ahilya-3(NCR -7) & Selection from S 69-96 & $\mathrm{CZ}$ & 1996 & ICAR, Indore \\
\hline 68 & MACS -450 & Bragg $\times$ MACS 111 & SZ & 1999 & MACS, Pune \\
\hline 69 & JS-90-41 & PS73-7 $\times$ Hark & $\mathrm{CZ}$ & 1999 & JNKVV, Jabalpur (MP) \\
\hline 70 & Prasad (MAUS -32) & Selection from JS 80-21 & SZ & 2000 & MAUS, Parbhani \\
\hline 71 & $\begin{array}{l}\text { Parbhani Sona } \\
\text { (MAUS- 47) }\end{array}$ & PS73-7 $\times$ Hark & $\mathrm{CZ}$ & 2000 & ICAR, Indore \\
\hline 72 & Pant soybean 1092 & PK $327 \times$ PK416 & NPZ & 1999 & GBPUA \& T, Pant Nagar \\
\hline 73 & LSb 1 & Selection from MACS 330 & $\mathrm{SZ}$ & 2001 & ANGRAU Hyderabad \\
\hline 74 & Indira soya -9 & Secondary selection from JS 80-21 & $\mathrm{CZ}$ & 1999 & IGKVV, Raipur \\
\hline 75 & $\begin{array}{l}\text { Hara soy (HIMSO - } \\
1563)\end{array}$ & $($ Ankur $\times$ Himso 330$) \times$ Bragg & NHZ & 2001 & CSKHPKV, Palampur- (HP.) \\
\hline 76 & Ahilya-4 (NCR -37) & Gaurav $\times$ Punjab 1 & $\mathrm{CZ}$ & 2001 & DSR Indore \\
\hline 77 & $\begin{array}{l}\text { Samrudhi (MAUS } \\
\text { 71) }\end{array}$ & JS 71-5 × JS 87-38 & $\mathrm{CZ}$ & 2002 & MAUS, Parbhani \\
\hline 78 & $\begin{array}{l}\text { Prtishta (MAUS-61- } \\
\text { 2) }\end{array}$ & JS80-21 X KB-60 & $\mathrm{CZ}$ & 2002 & MAUS, Parbhani \\
\hline 79 & $\begin{array}{l}\text { Pratikar (MAUS- } \\
61 \text { ) }\end{array}$ & JS71-1 × PK-73-94 & $\mathrm{CZ}$ & 2002 & MAUS, Parbhani \\
\hline 80 & $\begin{array}{l}\text { Pratap soya (RAUS } \\
\text { 5) }\end{array}$ & PUSA 16 x JS 335 & $\mathrm{CZ}$ & 2007 & MPUA,Udaipur \\
\hline 81 & MAUS 81(Shakti) & KB74 × JS335 & $\mathrm{SZ}$ & 2003 & MAUS,Parbhani \\
\hline
\end{tabular}




\begin{tabular}{|l|l|l|l|l|l|}
\hline $\mathbf{8 2}$ & Pant soybean 1241 & PK $1039 \times$ PK327 & NPZ & 2003 & GBPUA\&T, Pant Nagar \\
\hline $\mathbf{8 3}$ & TAMS-38 & Monetta $\times$ PK472 & SZ & 2004 & PDKV, Amravati \\
\hline $\mathbf{8 4}$ & SL 525 & PK 416 $\times$ PK1023 & NPZ & 2007 & PAU, Ludhiana \\
\hline $\mathbf{8 5}$ & Co-3 (Co soya) & UGM $99 \times$ JS335 & SZ & 2005 & TNAU, Coimbatore \\
\hline $\mathbf{8 6}$ & PRS -1 & Selection from germplasm & NPZ & 2009 & GBPUA\&, Pant Nagar \\
\hline $\mathbf{8 7}$ & $\begin{array}{l}\text { Pusa } 97-12 \text { (DS-97- } \\
\text { 12) }\end{array}$ & Mutant of DS74 & NPZ & 2007 & IARI, New Delhi \\
\hline $\mathbf{8 8}$ & JS-95-60 & Selection from PS 73-22 & CZ & 2007 & JNKVV, Jabalpur \\
\hline $\mathbf{8 9}$ & PS 1347 & PS $1024 \times$ PK472 & NPZ & 2006 & GBPUA\&T, Pant Nagar \\
\hline $\mathbf{9 0}$ & Pusa-98-14 & Bragg $\times$ DS 93-MM-39 & NPZ & 2006 & IARI, New Delhi \\
\hline $\mathbf{9 1}$ & $\begin{array}{l}\text { RKS } 18(\text { Pratap soya } \\
\text {-2) }\end{array}$ & MACS $450 \times$ Monetta & CZ & 2007 & MPUA, Udaipur \\
\hline $\mathbf{9 2}$ & TAMS -98 -21 & Mutant of JS80-21 & SZ & 2007 & PDKV, Akola \\
\hline $\mathbf{9 3}$ & PS -1225 & PK515 $\times$ PK327 & NPZ & 2007 & GBPUA\&T, Pant Nagar \\
\hline $\mathbf{9 4}$ & JS-97-52 & PK $327 \times$ L 129 & CZ & 2008 & $\begin{array}{l}\text { DSR, Indore and JNKVV, } \\
\text { Jabalpur }\end{array}$ \\
\hline $\mathbf{9 5}$ & SL 688 & PK416 $\times$ SL317 & NPZ & 2008 & PAU, Ludhiana \\
\hline $\mathbf{9 6}$ & PK -471 & Hardee $\times$ Punjab1 & SZ & 1988 & VNMKV, Parbhani \\
\hline
\end{tabular}

NHZ: Northern Hill Zone, NPZ:Northern Plain Zone, NEZ: Northern Eastern Zone, CZ: Central Zone, SZ: Southern Zone. RVSVV: Rajmata Vijayaraje Scindia Krishi Vishwavidyalaya, CSKHPKV: Chaudhary Sarwan Kumar Himachal Pradesh University, JNKVV: Jawaharlal Nehru Krishi Vishva Vidhyalya, VPKAS: Vivekananda Parvatiya Krishi Anusandhan Sansthan, MPKV: Mahatma Phule Krishi Vidyapeeth, GKVK: Gandhi Krishi Vignana Kendra, VNMKV: Vasntrao Naik Marathwada Krishi Vidyapeeth, IARI: Indian Agricultural Research Institute, PAU: Punjab Agricultural University, ICAR: Indian Council of Agricultural Research, TNAU: Tamil Nadu Agricultural University, MAUS: Marathwada Agricultural University, GBPU\&T: G.B Pant University of Agriculture and Technology, MACS: Maharashtra Academy for Cultivation of Sciences, IASRI: Indian Agricultural Statistics Research Institute, ANGRAU: Acharya N.G Ranga Agriculture University, IGKVV: Indian Gandhi Krishi Viswavidyalaya, MPUA: Maharana Pratap University of Agriculture and Technology, PDKV: Panjabrao Deshmukh Krishi Vidyapeeth, DSR: Directorate of Soybean Research

Table.2 List of SSR primers used for genetic diversity estimation and cultivar identification in soybean [Glycine $\max (\mathrm{L}$.$) Merrill]$

\begin{tabular}{|l|l|c|c|c|l|l|}
\hline $\begin{array}{l}\text { S. } \\
\text { No. }\end{array}$ & $\begin{array}{l}\text { Marker } \\
\text { name }\end{array}$ & $\begin{array}{c}\text { Linkage } \\
\text { group }\end{array}$ & $\begin{array}{c}\text { Chromo } \\
\text { some } \\
\text { number }\end{array}$ & Repeat motif & Forward primer & Reverse primer \\
\hline 1 & SAT 185 & G & 18 & $($ AT) 31 & GCGGCTGGAGAAAACCTTTTATG & $\begin{array}{l}\text { GCGAATAAAAACCGAGAATGA } \\
\text { TTT }\end{array}$ \\
\hline 2 & SAT 366 & J & 16 & $($ AT $) 8$ & $\begin{array}{l}\text { GCGGCACAAGAACAGAGGAAAC } \\
\text { TATT }\end{array}$ & $\begin{array}{l}\text { GCGGACATGGTACATCTATATT } \\
\text { ACGAGTATT }\end{array}$ \\
\hline 3 & SATT002 & D2 & 17 & $($ ATT $) 25$ & TGTGGGTAAAATAGATAAAAAT & TCATTTTGAATCGTTGAA \\
\hline 4 & SATT005 & D1b & 2 & (ATT) 19 & $\begin{array}{l}\text { TATCCTAGAGAAGAACTAAAAA } \\
\text { A }\end{array}$ & GTCGATTAGGCTTGAAATA \\
\hline 5 & SATT030 & F & 13 & (ATT) 21 & AAAAAGTGAACCAAGCC & TCTTAAATCTTATGTTGATGC \\
\hline 6 & SATT038 & G & 18 & (ATT) 17 & $\begin{array}{l}\text { GGGAATCTTTTTTTCTTTCTATTA } \\
\text { AGTT }\end{array}$ & $\begin{array}{l}\text { GGGCATTGAAATGGTTTTAGTC } \\
\text { A }\end{array}$ \\
\hline 7 & SATT082 & D2 & 17 & (ATT) 13 & AATTCATTTAGGGAGTTGAT & CTAGCCAATGTCATATGACT \\
\hline
\end{tabular}


Int.J.Curr.Microbiol.App.Sci (2020) 9(4): 449-465

\begin{tabular}{|c|c|c|c|c|c|c|}
\hline 8 & SATT173 & $\mathrm{O}$ & 10 & $(\mathrm{ATT}) 18$ & TGCGCCATTTATTCTTCA & AAGCGAAATCACCTCCTCT \\
\hline 9 & SATT177 & A2 & 8 & $(\mathrm{ATT}) 16$ & CGTTTCATTCCCATGCCAATA & CCCGCATCTTTTTCAACCAC \\
\hline 10 & $\begin{array}{l}\text { GlySATT } \\
180\end{array}$ & $\mathrm{C} 1$ & 4 & $(\mathrm{ATT}) 16$ & TCGCGTTTGTCAGC & TTGATTGAAACCCAACTA \\
\hline 11 & SATT181 & $\mathrm{H}$ & 12 & (ATT) 18 & TGGCTAGCAGATTGACA & GGAGCATAGCTGTTAGGA \\
\hline 12 & SATT183 & $\mathrm{J}$ & 16 & $(\mathrm{ATT}) 13$ & TAGGTCCCAGAATTTCATTG & CACCAACCAGCACAAAA \\
\hline 13 & $\begin{array}{l}\text { GlySATT } \\
184\end{array}$ & D1a & 1 & $(\mathrm{ATT}) 13$ & $\begin{array}{l}\text { GCGCTATGTAGATTATCCAAATT } \\
\text { ACGC }\end{array}$ & GCCACTTACTGTTACTCAT \\
\hline 14 & SATT197 & B1 & 11 & $(\mathrm{ATT}) 20$ & CACTGCTTTTTCCССТCTCT & $\begin{array}{l}\text { AAGATACCCCCAACATTATTTG } \\
\text { TAA }\end{array}$ \\
\hline 15 & SATT243 & $\mathrm{O}$ & 10 & $(\mathrm{ATT}) 17$ & $\begin{array}{l}\text { GCGCATTGCACATTAGGTTTTCT } \\
\text { GTT }\end{array}$ & $\begin{array}{l}\text { GCGGTAAGATCACGCCATTATT } \\
\text { TAAGA }\end{array}$ \\
\hline 16 & SATT244 & $\mathbf{J}$ & 16 & (ATT)27 & $\begin{array}{l}\text { GCGCCCCATATGTTTAAATTATA } \\
\text { TGGAG }\end{array}$ & $\begin{array}{l}\text { GCGATGGGGATATTTTCTTTAT } \\
\text { TATCAG }\end{array}$ \\
\hline 17 & SATT245 & M & 7 & $\begin{array}{l}\text { (ATT) } 13(\text { ATG } \\
) 15\end{array}$ & AACGGGAGTAGGACATTTTATT & $\begin{array}{l}\text { GCGCCTCCTGAATTTCAAAGAA } \\
\text { TGAAGA }\end{array}$ \\
\hline 18 & SATT250 & M & 7 & $(\mathrm{ATT}) 16$ & CGCCAGCTAGCTAGTCTCAT & $\begin{array}{l}\text { AATTTGCTCCAGTGTTTTAAGT } \\
\text { TT }\end{array}$ \\
\hline 19 & SATT264 & K & 9 & $(\mathrm{ATT}) 14$ & CCTTTTGACAATTATGGCATATA & $\begin{array}{l}\text { GCATAGAAGGGCATCATTCAG } \\
\text { AT }\end{array}$ \\
\hline 20 & $\begin{array}{l}\text { GlySATT } \\
267\end{array}$ & D1a & 1 & $(\mathrm{ATT}) 16$ & CCGGTCTGACCTATTCTCAT & CACGGCGTATTTTTATTTTG \\
\hline 21 & SATT268 & $\mathrm{E}$ & 15 & $(\mathrm{ATT}) 17$ & $\begin{array}{l}\text { TCAGGGGTGGACCTATATAAAA } \\
\text { TA }\end{array}$ & CAGTGGTGGCAGATGTAGAA \\
\hline 22 & $\begin{array}{l}\text { GlySATT } \\
277\end{array}$ & $\mathrm{C} 2$ & 6 & (ATT)40 & GGTGGTGGCGGGTTACTATTACT & $\begin{array}{l}\text { CCACGCTTCAGTTGATTCTTAC } \\
\text { A }\end{array}$ \\
\hline 23 & $\begin{array}{l}\text { GlySATT } \\
279\end{array}$ & $\mathrm{H}$ & 12 & $(\mathrm{ATT}) 28$ & $\begin{array}{l}\text { GCGCAAAAGGACGCCCACCAAT } \\
\text { AG }\end{array}$ & $\begin{array}{l}\text { GCGGTGATCGGATGTTATAGTT } \\
\text { TCAG }\end{array}$ \\
\hline 24 & SATT285 & $\mathbf{J}$ & 16 & $(\mathrm{ATT}) 19$ & $\begin{array}{l}\text { GCGACATATTGCATTAAAAACAT } \\
\text { ACTT }\end{array}$ & $\begin{array}{l}\text { GCGGACTAATTCTATTTTACAC } \\
\text { CAACAAC }\end{array}$ \\
\hline 25 & SATT286 & $\mathrm{C} 2$ & 6 & $(\mathrm{ATT}) 17$ & GCGGCGTTAATTTATGCCGGAAA & $\begin{array}{l}\text { GCGTTTGGTCTAGAATAGTTCT } \\
\text { CA }\end{array}$ \\
\hline 26 & SATT288 & G & 18 & $(\mathrm{ATT}) 17$ & $\begin{array}{l}\text { GCGGGGTGATTTAGTGTTTGACA } \\
\text { CCT }\end{array}$ & $\begin{array}{l}\text { GCGCTTATAATTAAGAGCAAA } \\
\text { AGAAG }\end{array}$ \\
\hline 27 & $\begin{array}{l}\text { GlySATT } \\
300\end{array}$ & A1 & 5 & $(\mathrm{ATT}) 19$ & GCGCCCACACAACCTTTAATCTT & GCGGCGACTGTTAACGTGTC \\
\hline 28 & SATT308 & M & 7 & $(\mathrm{ATT}) 21$ & $\begin{array}{l}\text { GCGTTAAGGTTGGCAGGGTGGA } \\
\text { AGTG }\end{array}$ & $\begin{array}{l}\text { GCGCAGCTTTATACAAAAATC } \\
\text { AACAA }\end{array}$ \\
\hline 29 & SATT309 & G & 18 & $(\mathrm{ATT}) 13$ & GCGCCTTCAAATTGGCGTCTT & $\begin{array}{l}\text { GCGCCTTAAATAAAACCCGAA } \\
\text { ACT }\end{array}$ \\
\hline 30 & SATT324 & G & 18 & (ATT)19 & GTTCCCAGGTCCCACCATCTATG & GCGTTTCTTTTATACCTTCAAG \\
\hline 31 & SATT335 & $\mathrm{F}$ & 13 & $(\mathrm{ATT}) 12$ & CAAGCTCAAGCCTCACACAT & TGACCAGAGTCCAAAGTTCATC \\
\hline 32 & SATT337 & K & 9 & (ATT) 19 & $\begin{array}{l}\text { GCGTAAATCTGATATATGTTACC } \\
\text { ACTGA }\end{array}$ & $\begin{array}{l}\text { GCGTAATACGCAAAACATAAT } \\
\text { TAGCCTA }\end{array}$ \\
\hline 33 & $\begin{array}{l}\text { GlySATT } \\
345\end{array}$ & $\mathrm{O}$ & 10 & (ATT)27 & $\begin{array}{l}\text { CCCCTATTTCAAGAGAATAAGG } \\
\text { AA }\end{array}$ & $\begin{array}{l}\text { CCATGCTCTACATCTTCATCAT } \\
\text { C }\end{array}$ \\
\hline
\end{tabular}


Int.J.Curr.Microbiol.App.Sci (2020) 9(4): 449-465

\begin{tabular}{|c|c|c|c|c|c|c|}
\hline 34 & SATT346 & $\mathrm{F}$ & 13 & (ATT) 18 & CGTCGCCATCACTATGAGAA & $\begin{array}{l}\text { CCATCTTGAGCAGAGTTTGAAG } \\
\text { TT }\end{array}$ \\
\hline 35 & SATT373 & $\mathrm{L}$ & 19 & $(\mathrm{ATT}) 21$ & TCCGCGAGATAAATTCGTAAAAT & $\begin{array}{l}\text { GGCCAGATACCCAAGTTGTACT } \\
\text { TGT }\end{array}$ \\
\hline 36 & SATT406 & $\mathrm{J}$ & 16 & (ATT)31 & GCGTGAGCATTTTTGTTT & TGACGGGTTTAATAGCAT \\
\hline 37 & SATT431 & $\mathrm{J}$ & 16 & $(\mathrm{ATT}) 21$ & GCGTGGCACCCTTGATAAATAA & $\begin{array}{l}\text { GCGCACGAAAGTTTTTCTGTAA } \\
\text { CA }\end{array}$ \\
\hline 38 & SATT440 & I & 20 & $(\mathrm{ATT}) 14$ & TGAGAACGTTTGAAAAGAGAT & $\begin{array}{l}\text { GAAGAGATTAAGCATAAAGAA } \\
\text { TACTT }\end{array}$ \\
\hline 39 & $\begin{array}{l}\text { GlySATT } \\
449\end{array}$ & A1 & 5 & $(\mathrm{ATT}) 21$ & $\begin{array}{l}\text { GCGTGCTTCTTATATTAGGTGTT } \\
\text { AGT }\end{array}$ & GCGCATTGGAGTTTTTGCTTTT \\
\hline 40 & $\begin{array}{l}\text { GlySATT } \\
453\end{array}$ & B1 & 11 & $(\mathrm{ATT}) 13$ & $\begin{array}{l}\text { GCGGAAAAAAAACAATAAACAA } \\
\text { CA }\end{array}$ & TAGTGGGGAAGGGAAGTTACC \\
\hline 41 & $\begin{array}{l}\text { GlySATT } \\
530\end{array}$ & $\mathrm{~N}$ & 3 & $(\mathrm{ATT}) 12$ & CATGCATATTGACTTCATTATT & $\begin{array}{l}\text { CCAAGCGGGTGAAGAGGTTTT } \\
\mathrm{T}\end{array}$ \\
\hline 42 & SATT534 & B2 & 14 & (ATT) 25 & СТССТССТGCGCAACAACAATA & GGGGGATCTAGGCCATGAC \\
\hline 43 & SATT562 & I & 20 & $(\mathrm{ATT}) 18$ & GCGGATTGACTGAGATGTTTAT & $\begin{array}{l}\text { GCGGCGGCAGGTTAAATGGAT } \\
\text { TGA }\end{array}$ \\
\hline 44 & $\begin{array}{l}\text { GlySATT } \\
577\end{array}$ & B2 & 14 & $(\mathrm{ATT}) 12$ & $\begin{array}{l}\text { CAAGCTTAAGTCTTGGTCTTCTC } \\
\mathrm{T}\end{array}$ & $\begin{array}{l}\text { GGCCTGACCCAAAACTAAGGG } \\
\text { AAGTG }\end{array}$ \\
\hline 45 & $\begin{array}{l}\text { GlySATT } \\
586\end{array}$ & $\mathrm{~F}$ & 13 & $(\mathrm{ATT}) 19$ & GCGGCCTCCAAACTCCAAGTAT & $\begin{array}{l}\text { GCGCCCAAATGATTAATCACTC } \\
\text { A }\end{array}$ \\
\hline 46 & $\begin{array}{l}\text { GlySATT } \\
588\end{array}$ & $\mathrm{~K}$ & 9 & $\begin{array}{l}(\mathrm{ATT}) 18(\mathrm{AT}) \\
10(\mathrm{CT}) 14\end{array}$ & $\begin{array}{l}\text { GCTGCATATCCACTCTCATTGAC } \\
\mathrm{T}\end{array}$ & $\begin{array}{l}\text { GAGCCAAAACCAAAGTGAAGA } \\
\text { AC }\end{array}$ \\
\hline 47 & SATT590 & M & 7 & (ATT)26 & $\begin{array}{l}\text { GCGCGCATTTTTTAAGTTAATGT } \\
\text { TCT }\end{array}$ & $\begin{array}{l}\text { GCGCGAGTTAGCGAATTATTTG } \\
\text { TC }\end{array}$ \\
\hline 48 & $\begin{array}{l}\text { SOYPRP- } \\
1\end{array}$ & - & - & $(\mathrm{TAT}) 20$ & CGTGCCAAATTACATCA & TGATGGGAACAAGTACATAA \\
\hline 49 & $\begin{array}{l}\text { SOYSAT } \\
\mathrm{T}-005\end{array}$ & $\mathrm{D} 1 \mathrm{~b}+\mathrm{W}$ & - & $(\mathrm{ATT}) 19$ & $\begin{array}{l}\text { TATATCCTAGAGAAGAACTAAA } \\
\text { AAA }\end{array}$ & $\begin{array}{l}\text { GTCGATTAGGCTTGAAATAAT } \\
\text { AC }\end{array}$ \\
\hline
\end{tabular}

Table.3 SSR primers and their characteristics for diversity assessment and cultivar identification in soybean [Glycine max (L.) Merrill]

\begin{tabular}{|l|l|l|l|l|l|l|l|l|l|l|l|}
\hline $\begin{array}{l}\text { S. } \\
\text { No. }\end{array}$ & Primer name & $\begin{array}{l}\text { Size } \\
\text { range } \\
\text { (bp) }\end{array}$ & NSB & NPB & PPB & $\begin{array}{l}\text { Gene } \\
\text { diversity }\end{array}$ & RP & MI & $\begin{array}{l}\text { Unique } \\
\text { band size } \\
\text { (Cultivar) }\end{array}$ & PIC $_{\mathbf{e}}$ \\
\hline $\mathbf{1}$ & SATT-268 & $300-360$ & 4 & 4 & $100 \%$ & 0.72 & 2 & 2.884 & - & 0.67 & 0.72 \\
\hline $\mathbf{2}$ & SATT-002 & $120-150$ & 5 & 5 & $100 \%$ & 0.70 & 2 & 3.488 & - & 0.65 & 0.70 \\
\hline $\mathbf{3}$ & SATT-038 & $170-480$ & 7 & 7 & $100 \%$ & 0.73 & 2 & 5.082 & $470 b p($ PK-471) & 0.69 & 0.73 \\
\hline $\mathbf{4}$ & SATT-590 & $260-340$ & 4 & 4 & $100 \%$ & 0.65 & 2 & 2.612 & - & 0.61 & 0.67 \\
\hline $\mathbf{5}$ & SATT-082 & $100-130$ & 4 & 4 & $100 \%$ & 0.68 & 2 & 2.713 & - & 0.62 & 0.68 \\
\hline $\mathbf{6}$ & SATT-286 & $200-230$ & 4 & 4 & $100 \%$ & 0.66 & 2 & 2.629 & - & 0.55 & 0.59 \\
\hline $\mathbf{7}$ & SATT-197 & $140-180$ & 5 & 5 & $100 \%$ & 0.59 & 2 & 2.964 & - & 0.51 & 0.59 \\
\hline $\mathbf{8}$ & SATT-005 & $140-190$ & 6 & 6 & $100 \%$ & 0.71 & 2 & 4.306 & $140 \mathrm{bp}($ JS-97-52) & 0.67 & 0.72 \\
\hline $\mathbf{9}$ & SATT-335 & $140-250$ & 4 & 4 & $100 \%$ & 0.62 & 2 & 2.499 & - & 0.55 & 0.63 \\
\hline $\mathbf{1 0}$ & SATT-285 & $200-240$ & 3 & 3 & $100 \%$ & 0.46 & 2 & 1.392 & - & 0.33 & 0.39 \\
\hline $\mathbf{1 1}$ & SOY SATT- & $150-190$ & 5 & 5 & $100 \%$ & 0.72 & 2 & 3.603 & - & 0.67 & 0.72 \\
\hline
\end{tabular}




\begin{tabular}{|c|c|c|c|c|c|c|c|c|c|c|c|}
\hline & 005 & & & & & & & & & & \\
\hline 12 & SOYPRP -1 & $120-150$ & 4 & 4 & $100 \%$ & 0.54 & 2 & 2.146 & - & 0.47 & 0.54 \\
\hline 13 & Gly SATT-267 & $230-250$ & 3 & 3 & $100 \%$ & 0.62 & 2 & 1.872 & - & 0.51 & 0.57 \\
\hline 14 & Gly SATT-279 & $180-200$ & 3 & 3 & $100 \%$ & 0.61 & 2 & 2.451 & - & 0.43 & 0.51 \\
\hline 15 & Gly SATT-577 & $80-130$ & 5 & 5 & $100 \%$ & 0.76 & 2 & 3.815 & - & 0.72 & 0.76 \\
\hline 16 & Gly SATT-586 & $170-230$ & 7 & 7 & $100 \%$ & 0.69 & 2 & 4.853 & $\begin{array}{l}\text { 170bp (Lee), } \\
\text { 230bp (MACS-58) }\end{array}$ & 0.66 & 0.69 \\
\hline 17 & $\begin{array}{l}\text { SOY SATT- } \\
183\end{array}$ & $200-270$ & 6 & 6 & $100 \%$ & 0.78 & 2 & 4.662 & - & 0.66 & 0.69 \\
\hline 18 & SATT-373 & $200-290$ & 6 & 6 & $100 \%$ & 0.79 & 2 & 4.728 & - & 0.76 & 0.79 \\
\hline 19 & SATT-177 & $100-200$ & 6 & 6 & $100 \%$ & 0.77 & 2 & 4.647 & - & 0.74 & 0.78 \\
\hline 20 & Gly SATT-453 & $240-280$ & 4 & 4 & $100 \%$ & 0.68 & 2 & 3.416 & - & 0.60 & 0.64 \\
\hline 21 & SATT-534 & $160-190$ & 5 & 5 & $100 \%$ & 0.73 & 2 & 3.657 & 175bp (Co-3) & 0.68 & 0.73 \\
\hline 22 & SATT-244 & $120-180$ & 6 & 6 & $100 \%$ & 0.65 & 2 & 3.88 & - & 0.53 & 0.60 \\
\hline 23 & SATT-346 & $170-210$ & 5 & 4 & $80 \%$ & 0.65 & 2 & 2.58 & - & 0.59 & 0.64 \\
\hline 24 & SATT-309 & $120-150$ & 4 & 4 & $100 \%$ & 0.62 & 2 & 2.488 & 150bp (Bragg) & 0.54 & 0.62 \\
\hline 25 & SATT-173 & $150-220$ & 6 & 6 & $100 \%$ & 0.75 & 2 & 4.519 & - & 0.72 & 0.75 \\
\hline 26 & Gly SATT-588 & $120-190$ & 5 & 5 & $100 \%$ & 0.73 & 2 & 3.668 & - & 0.69 & 0.73 \\
\hline 27 & SATT-243 & $270-300$ & 3 & 3 & $100 \%$ & 0.44 & 2 & 1.348 & - & 0.38 & 0.45 \\
\hline 28 & SAT-366 & $190-200$ & 2 & 2 & $100 \%$ & 0.60 & 2 & 1.804 & - & 0.13 & 0.14 \\
\hline 29 & SATT-245 & $190-210$ & 3 & 3 & $100 \%$ & 0.64 & 2 & 1.917 & - & 0.49 & 0.57 \\
\hline 30 & SATT-264 & $190-220$ & 4 & 4 & $100 \%$ & 0.66 & 2 & 2.621 & - & 0.59 & 0.66 \\
\hline 31 & SATT-288 & $190-220$ & 4 & 4 & $100 \%$ & 0.68 & 2 & 2.737 & - & 0.63 & 0.68 \\
\hline 34 & SATT-406 & $240-320$ & 5 & 5 & $100 \%$ & 0.72 & 2 & 3.607 & - & 0.68 & 0.72 \\
\hline 35 & SATT-440 & $170-220$ & 6 & 6 & $100 \%$ & 0.70 & 2.23 & 4.174 & - & 0.72 & 0.76 \\
\hline 36 & SATT-562 & $190-220$ & 4 & 4 & $100 \%$ & 0.70 & 2 & 2.814 & - & 0.40 & 0.42 \\
\hline 37 & SATT-030 & $130-170$ & 5 & 5 & $100 \%$ & 0.74 & 2 & 3.690 & - & 0.69 & 0.74 \\
\hline 38 & SATT-181 & $180-210$ & 4 & 4 & $100 \%$ & 0.73 & 2 & 2.94 & - & 0.68 & 0.74 \\
\hline 39 & SATT-324 & $220-240$ & 3 & 3 & $100 \%$ & 0.66 & 2 & 1.968 & - & 0.58 & 0.66 \\
\hline 40 & SATT-250 & $180-210$ & 4 & 4 & $100 \%$ & 0.59 & 2 & 1.761 & - & 0.57 & 0.62 \\
\hline 41 & SATT-431 & $180-230$ & 5 & 5 & $100 \%$ & 0.78 & 2 & 3.903 & - & 0.74 & 0.78 \\
\hline 42 & SAT-185 & $200-270$ & 6 & 6 & $100 \%$ & 0.77 & 2 & 4.624 & - & 0.74 & 0.77 \\
\hline 43 & GlySATT-277 & $170-250$ & 6 & 6 & $100 \%$ & 0.76 & 2 & 4.570 & - & 0.73 & 0.76 \\
\hline 44 & Gly SATT-184 & $140-180$ & 4 & 4 & $100 \%$ & 0.70 & 2 & 2.787 & - & 0.64 & 0.70 \\
\hline 45 & Gly SATT-449 & $230-280$ & 6 & 6 & $100 \%$ & 0.80 & 2 & 4.777 & - & 0.77 & 0.80 \\
\hline 46 & Gly SATT-300 & $250-270$ & 3 & 3 & $100 \%$ & 0.64 & 2 & 1.932 & - & 0.46 & 0.52 \\
\hline 47 & Gly SATT-530 & $190-250$ & 6 & 6 & $100 \%$ & 0.78 & 2 & 4.651 & - & 0.74 & 0.78 \\
\hline 48 & Gly SATT-345 & $210-260$ & 6 & 6 & $100 \%$ & 0.73 & 2 & 4.388 & 210bp (KHsb-2) & 0.69 & 0.73 \\
\hline 49 & Gly SATT-180 & $230-290$ & 6 & 6 & $100 \%$ & 0.73 & 2 & 4.364 & - & 0.69 & 0.73 \\
\hline
\end{tabular}

NSB: Number of scored band, NMB: Number of monomorphic bands, NPB: Number of polymorphic band, PPB:

Percentage of polymorphic band, PIC: Polymorphism information content, RP: Resolving power, MI: Marker index, and $\mathrm{H}_{\mathrm{e}}$ : heterozygosity 
Figure.1 PCR amplification profile of 96 soybean [Glycine max (L.) Merrill] cultivars generated using SSR marker SATT 268. The numbers 1 through 96 on top of each lane corresponds to the cultivar name as indicated in Table 1. $\mathrm{M}$ is 100-base pair molecular weight standard
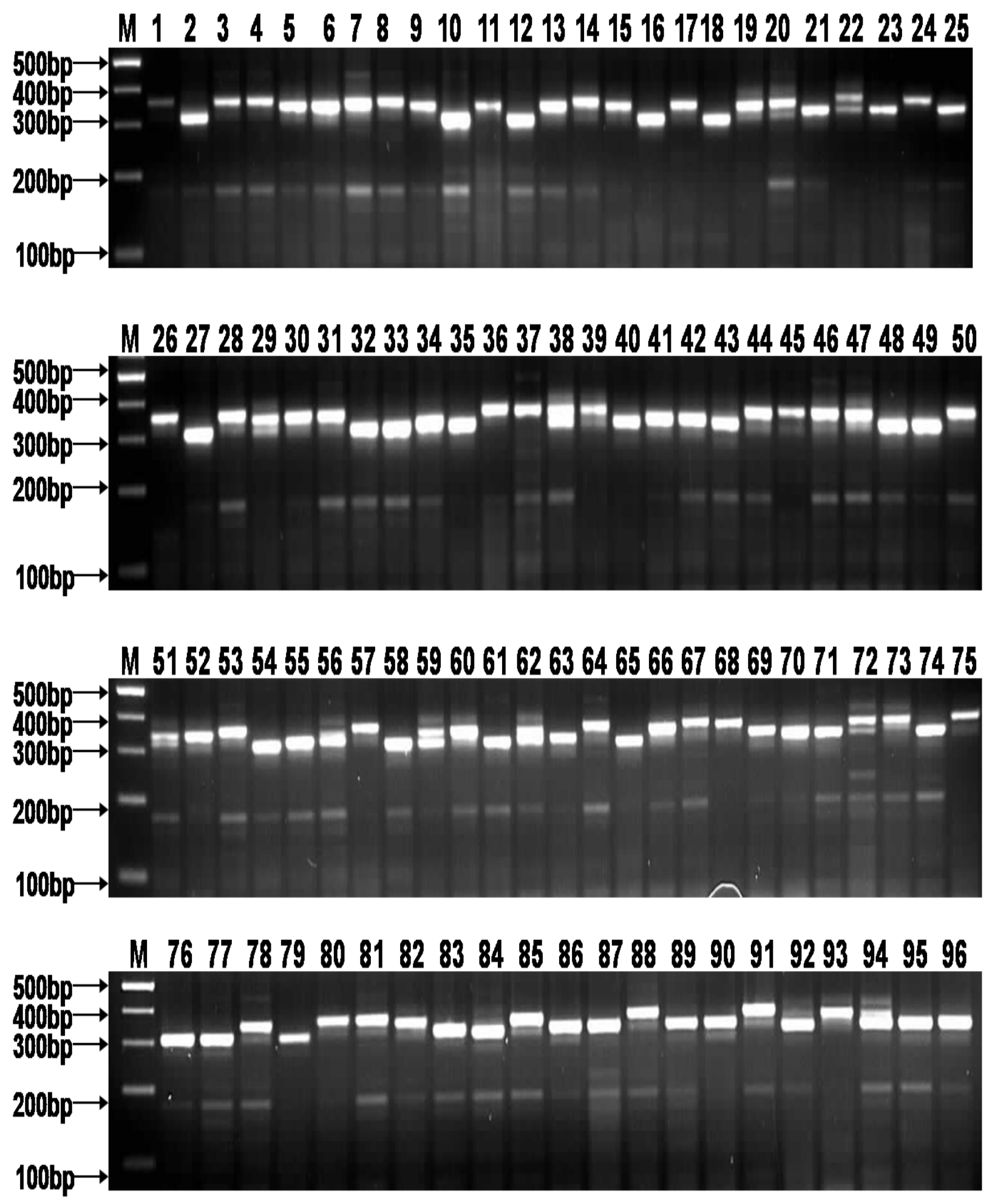
Figure.2 Dendrogram depicting genetic relationships (Simple Matching coefficient) among 96 soybean [Glycine max (L.) Merrill] cultivars generated using

230 markers from 49 SSR primer-pairs

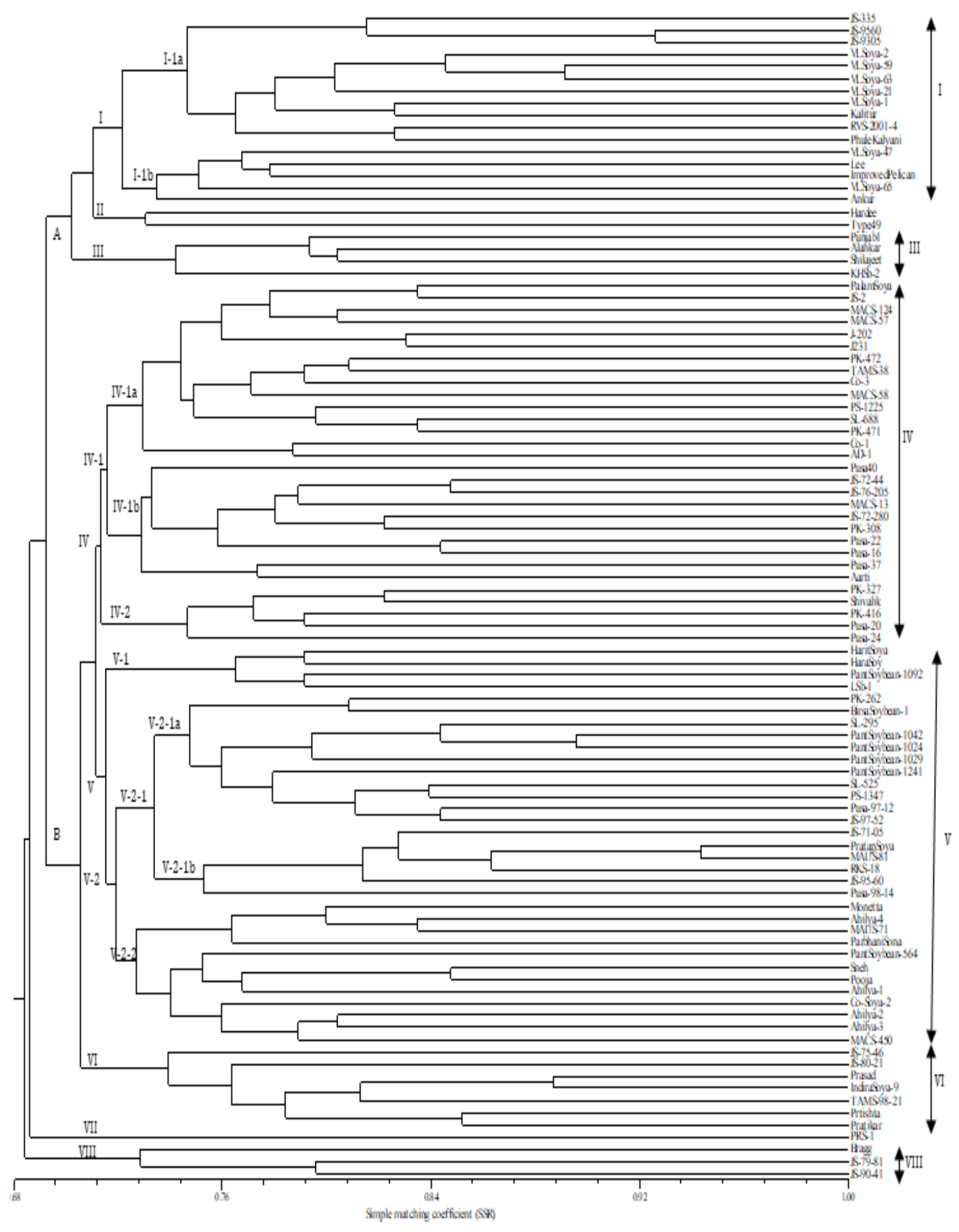


The present study revealed the genetic diversity of 96 Indian soybean cultivars using SSR markers. The data revealed that $29 \%$ genetic dissimilarity existed in the material. Cluster analysis showed the genetic relationships among the cultivars and diverse cultivars as parents from different clusters can be selected. The results obtained in our study could be of practical application in breeding of soybean to produce better cultivars with high yielding characters. SSR markers were quite efficient in discriminating the cultivars from one another and the presence of unique bands highlighted the importance of their utility in IPR protection.

\section{Acknowledgements}

The authors are grateful to Director, ICARNational Bureau of Plant Genetic Resources to provide the laboratory facilities to undertake the work. Financial support received from Indian Council of Agricultural Research in terms of institutional project is duly acknowledged.

\section{References}

Akkaya, M. S., Shoemaker, R. C., Specht, J. E., Bhagwat, A. A., and Cregan, P. B. 1995. Integration of simple sequence repeat DNA markers into a soybean linkage map. Crop Science 35: 1439-1445.

Bisen, A., Khare, D., Nair, P., and Tripathi, N. 2015. SSR analysis of 38 genotypes of soybean (Glycine Max (L.) Merr.) genetic diversity in India. Physiology and Molecular Biology of Plants. 21: 109115.

Botstein, D., White, R. L., Skolnick, M. and Davis, R. W. 1980. Construction of a genetic linkage map in man using restriction fragment length polymorphisms. American Journal of Human Genetics. 32: 314-331.

Chandra, V., Pant, U., Bhajan, R. and Singh,
A.K. 2013. Studies on genetic diversity among Alternaria blight tolerant Indian mustard genotypes using SSR markers. The Bioscan. 8:1431-1435

Chauhan, D. K., Bhat, J. A., Thakur, A. K., Kumari, S., Hussain, Z., and Satyawathi, C. T. 2015. Molecular characterization and genetic diversity assessment in soybean [Glycine max (L.) Merr.] cultivars using SSR markers. Indian Journal of Biotechnology. 14: 504-510.

Cho, Y. H., M. S. Yoon, J. Lee, H. Y. Baek, C. Y. Kim, T. S. Kim, E. G. Cho, and H. B. Lee. 2006: Diversity and geographical relationships by SSR marker in subgenus Soja originated from Korea. Korean J. Crop Sci. 51: 239-247.

Chotiyarnwong, O., Chatwachirawong, P., Chanprame, S., and Srinives, P. 2007. Evaluation of genetic diversity in Thai indigenous and recommended soybean cultivars by SSR markers. Thai Journal of Agricultural Science. 40: 119-126.

Chung, G., and Singh, R. J. 2008. Broadening the genetic base of soybean: a multidisciplinary approach. Critical Reviews in Plant Sciences. 27: 295-341.

Diwan, N., and Cregan, P. B. 1997. Automated sizing of fluorescent-labeled simple sequence repeat (SSR) markers to assay genetic variation in soybean. Theoretical and Applied Genetics. 95: 723-733.

Ghosh, J., Ghosh, P.D., and Choudhury, P. R. 2014. An assessment of genetic relatedness between soybean [Glycine $\max ($ L.) Merrill] cultivars using SSR markers. American Journal of Plant Sciences. 5: 3089.

Gopinath, P., and Pavadai, P. 2015. Morphology and yield parameters and biochemical analysis of soybean [Glycine $\max (\mathrm{L}$.$) Merrill) using gamma rays, EMS$ and DES treatment. International Letters of Natural Sciences. 35: 50-58.

Gupta, S.K., and Manjaya J.G. 2017. Genetic diversity and population structure of Indian soybean [Glycine Max (L.) Merr.] 
revealed by simple sequence repeat markers. J Crop Sci. Biotech. 20:221-231 DOI No. 10.1007/s12892-017-0023-0

Koutu, G.K., Shrivastava A., Singh Y., and Tiwari, S. 2019. Molecular characterization and genetic diversity assessment of soybean cultivars using SSR markers. Int. J. Curr. Microbiol. App. $\quad$ Sci., $\quad$ 8: $\quad 173-182$ https://doi.org/10.20546/ijcmas. 2019.804.018

Kumar, J., Verma, V., Goyal, A., Shahi, A.K., Sparoo, R., Sangwan, R.S., and Qazi, G.N. 2009. Genetic diversity analysis in Cymbopogon species using DNA markers. Plant Omics J. 2:20-29.

Kumawat, G., Singh, G., Gireesh, C., Shivakumar, M., Arya, M., Agarwal, D. K., and Husain, S. M. 2015. Molecular characterization and genetic diversity analysis of soybean (Glycine max (L.) Merr.) germplasm accessions in India. Physiology and Molecular Biology of Plants. 21: 101-107.

Lacape, J. M., Dessauw, D., Rajab, M., Noyer, J. L., and Hau, B. 2007. Microsatellite diversity in tetraploid Gossypium germplasm: assembling a highly informative genotyping set of cotton SSRs. Molecular Breeding. 19: 45-58.

Nawaz, M.A., Yang, S.H., Rehman, H.M., Baloch, F.S., Lee, J.D., Park, J.H., and Chung, G. 2017. Genetic diversity and population structure of Korean wild soybean (Glycine soja Sieb. and Zucc.) inferred from microsatellite markers. Biochemical Systematics and Ecology 71: 87-96

Pagar, T. A., Akhare, A. A., Gahukar, S. J., Khwaja, M. S., and Gawande, A. M. 2017. DNA fingerprinting of soybean (Glycine max L.) genotypes by using simple sequence repeats (SSR) markers. International Journal of Chemical Studies. 5: 674-679.

Powell, W., Machray, G. C., and Provan, J. 1996. Polymorphism revealed by simple sequence repeats. Trends in Plant Science. 1: 215-222.
Prevost, A., and Wilkinson, M. J. 1999. A new system of comparing PCR primers applied to ISSR fingerprinting of potato cultivars. Theoretical and Applied Genetics. 98: 107-112.

Rani, A., Kumar, V., Shukla, S., Jha, P. and Rawal, R. 2016. DNA barcoding of Indian soybean cultivars as constructed through SSR markers. Seed Sci. \& Technol. 44: 357-369. http://doi.org/10.15258/sst.2016.44.2.18

Rohlf F. James 2009. NTSYS-pc: Numerical taxonomy and multivariate analysis system version 2.2. Department of Ecology and Evolution State University of New York Stony Brook, NY 117945245 ISBN: 0-925031-31-3

Saghai-Maroof, M.A., Soliman, K.M., Jorgensen, R.A., and Allard, R.W. 1984. Ribosomal DNA spacer-length polymorphism in barley: Mendelian inheritance, chromosomal location, and population dynamics. Proc. Natl. Acad. Science. 81: 8014-8018.

Sahu, P., Khare, D., Tripathi, N., Shrivastava, A.N., and Saini, N. 2012. Molecular screening for disease resistance in soybean. J. Food Leg. 25:200- 205.

Singh, R. J., and Hymowitz, T. 1999. Soybean genetic resources and crop improvement. Genome. 42: 605-616.

Singh, R.K., Bhatia, V.S., Bhat, K.V., Mohapatra, T., Singh, N.K., Bansal, K.C., Koundal, and K.R. 2010. SSR and AFLP based genetic diversity of soybean germplasm differing in photoperiod sensitivity. Genet and Mol Bio. 33: 319324.

Song, Q.J., Marek, L.F., Shoemaker, R.C., Lark, K.G., Concibido, V.C., Delannay, X., Pecht, J.E.S., and Cregan, P.B. 2004. A new integrated genetic linkage map of the Soybean. Theor. Appl. Genet., 109: $122-128$.

Tantasawat, P., Trongchuen, J., Prajongjai, T., Jenweerawat, S., and Chaowiset, W. 2011. SSR analysis of soybean (Glycine $\max (\mathrm{L}$.$) Merr.) genetic relationships and$ cultivar Identification in Thailand. 
Australian Journal of Crop Science. $5: 283$.

Tautz, D., and Renz, M. 1984. Simple sequences are ubiquitous repetitive components of eukaryotic genomes. Nucleic Acids Research. 12: 4127-4138.

Velusamy, V., Toan, V. D., and Park, E. 2013. Evaluation of genetic diversity in wild soybean (Glycine soja Sieb. and Zucc.) accessions based on SSR markers. International Journal of Agronomy and Plant Production. 4: 1241-1246.

Vinu, V., Singh, N., Vasudev, S., Yadava, D.K., Kumar, S., Naresh, S., Bhat, S.R., and Prabhu, K.V. 2013. Assessment of genetic diversity in Brassica juncea (Brassicaceae) genotypes using phenotypic differences and SSR markers. Rev Biol Trop 61:1919-1934.

Vos, P., Hogers, R., Bleeker, M., Reijans, M., van de Lee, T., Hornes, M., Frijters ,A., Pot, J., Peleman, J., Kuiper, M. and Zabeau, M. 1995. AFLP: a new technique for DNA fingerprinting. Nucleic Acids Research. 23: 4407- 4414.

Wang, Y.H., Zhang, X.J. and Fan, S.J. 2015. Genetic diversity of wild soybean populations in Dongying, China, by simple sequence repeat analysis. Genetics and Molecular Research 14: 1161311623.

Williams, J. G., Kubelik, A. R., Livak, K. J., Rafalski, J. A., and Tingey, S. V. 1990. DNA polymorphisms amplified by arbitrary primers are useful as genetic markers. Nucleic Acids Research. 18: 6531-6535.

Yoon, M. S., Lee, J., Kim, C. Y., Kang, J. H., Cho, E. G., and Baek, H. J. 2009. DNA profiling and genetic diversity of Korean soybean [Glycine $\max$ (L.) Merrill] landraces by SSR markers. Euphytica. 165: 69-77.

Yun-Lai, G.A.O., Rong-Sheng, Z.H.U., ChunYan, L.I.U., Wen-Fu, L.I., Jiang, H.W., Can-Dong, L.I., and Qing-Shan, C.H.E.N. 2009. Constructing molecular identity for soybean cultivars from Heilongjiang Province, China. Acta Agronomica Sinica. 35: 211-218.

Zhao, H., Wang, Y., Xing, Fu, L., X., Yuan, C., Qi, G., Guo, J., and Dong, Y. 2018. The genetic diversity and geographic differentiation of the wild soybean in Northeast China based on nuclear microsatellite variation. Hindawi International Journal of Genomics: 2018, Article ID 8561458, 9 pageshttps://doi.org/10.1155/2018/85614 58.

Zietkiewicz, E., Rafalski, A., and Labuda, D. 1994. Genome fingerprinting by simple sequence repeat (SSR)-anchored polymerase chain reaction amplification. Genomics. 20: 176-183.

\section{How to cite this article:}

Shipra Deo, Anto James, Sanjeev Kumar Singh, C. B. Singh and Mukesh Kumar Rana. 2020. Molecular Diversity Analysis and Cultivar Identification using Simple Sequence Repeat (SSR) Markers in Soybean [Glycine Max (L.) Merrill]. Int.J.Curr.Microbiol.App.Sci. 9(04): 449-465. doi: https://doi.org/10.20546/ijcmas.2020.904.054 